\title{
Peer Reviewers
}

The number of submissions to Economic Botany is growing steadily, particularly due to international access via the internet, putting more and more pressure on our pool of talented reviewers. On behalf of the Society for Economic Botany, I extend my personal thanks to the men and women who agreed to peer reviewer manuscript submissions during 2012. The number in parentheses indicates multiple reviews. I apologize for any names I may have missed. I also give a special thanks to our Associate Editors, who do most of the real work of the journal, and who are frequently called upon to review additional manuscripts.

\author{
Alain Touwaide \\ Alan Hamilton \\ Allison L. Hopkins \\ Amin ul Haq Khan \\ Anthony Amend (2) \\ Bruce Benz \\ Bryan Hanson \\ Carlos Quiros \\ Charles R. Clement \\ Charles Peters \\ Charlie Shackleton \\ Christopher Gotz \\ Chunlin Long \\ Daniel E. Moerman (2) \\ David Lentz \\ Denise Stanley \\ Doyle McKey \\ Ergin Murat Altuner \\ Eugene Anderson (2)
}

\author{
Felix G. Coe \\ Freerk Wiersum \\ Gabriele Volpato \\ Geoffrey M. Wahungu \\ Hugo Joris de Boer \\ Huyin Huai \\ Ina Vandebroek \\ Ismuhan Potoglu Erkara \\ James Campbell Plowden \\ James Trappe \\ Jeffrey Ross-Ibarra \\ John Hart \\ Joost van Heerwaarden \\ Li Cheng-Sen \\ Marco Leonti \\ Mark Nesbitt \\ Marta Turok \\ Mary W. Eubanks \\ Mary L. Simon
}

\author{
Mary Theresa Bonhage- \\ Freund \\ Michael Nee \\ Nanci Ross (2) \\ Natalia Hanazaki \\ Niels Fold \\ Orou Gaoue \\ Peter Boyce \\ Rainer W. Bussmann \\ Scott Zona \\ Selena Ahmed \\ Simon Mayo \\ Stanford Zent (2) \\ Steve Siebert \\ Tamara Ticktin \\ Tom D. Dillehay \\ Victoria Reyes-Garcia (2) \\ Wendy Applequist (2) \\ Will McClatchey (3)
}

\footnotetext{
Published online 12 March 2013.
} 